\title{
The Lazy S Design: A Novel Skin Closure Design in Skin-Sparing Mastectomy for Implant-Based Breast Reconstruction
}

\author{
June-Kyu Kim, Yong Seong Kim \\ Department of Plastic and Reconstructive Surgery, Kangbuk Samsung Hospital, Sungkyunkwan University School of Medicine, Seoul, Korea
}

Preservation of the breast skin envelope during immediate implant-based breast reconstruction is important for producing symmetrical and natural-looking breasts. We propose the lazy $\mathrm{S}$ design for the closure of round-shaped wounds with the hope of improving the aesthetic outcomes and reducing the tension on the wound by preserving the skin. Additionally, the direction of tension is dispersed due to the shape of this design. Patients undergoing implant reconstruction after skin-sparing mastectomy may benefit from the lazy $\mathrm{S}$ design.

Keywords Mastectomy, subcutaneous / Mammoplasty / Breast implant / Reconstructive surgical procedures

\author{
Correspondence: June-Kyu Kim \\ Department of Plastic and \\ Reconstructive Surgery, Kangbuk \\ Samsung Hospital, Sungkyunkwan \\ University School of Medicine, 29 \\ Saemunan-ro, Jongno-gu, Seoul \\ 03181, Korea \\ Tel: +82-2-2001-2178 \\ Fax: +82-2-2001-2177 \\ E-mail: J7181.kim@samsung.net
}

Received: 8 Jul 2016 • Revised: 30 Mar 2017 • Accepted: 2 May 2017

pISSN: 2234-6163 • elSSN: 2234-6171 • https://doi.org/10.5999/aps.2017.44.4.344• Arch Plast Surg 2017;44:344-347

This article was presented as a poster at the 72nd Congress of the Korean Society of Plastic and Reconstructive Surgeons on November 7-9, 2014 in Seoul, Korea.

No potential conflict of interest relevant to this article was reported.

\section{INTRODUCTION}

Preservation of the breast skin envelope during immediate implant-based breast reconstruction is important for producing symmetrical and natural-looking breasts. The traditional fusiform excision design commonly used for the closure of the excised nipple-areolar complex (NAC) area after skin-sparing mastectomy (SSM) causes skin deficiency due to the large amount of skin that is resected. This causes visible asymmetry of the breast contour [1]. In particular, when direct-to-implant (DTI) breast reconstruction is performed after SSM, asymmetry is prominent because there is no tissue expansion process, unlike in 2-stage operations. In contrast, 2-stage operations can overcome skin deficiency to some extent. However, if it is possible to preserve the skin to an even greater extent during any of the reconstruction procedures, the skin expansion can be increased, which can lead to a better aesthetic outcome. We propose the lazy $S$ design for the closure of round-shaped wounds, with the hope of improving aesthetic outcomes and reducing the tension on the wound by preserving the skin.

\section{IDEA}

Type I SSM was performed as proposed by Carlson et al. [2]. 
Implant-based breast reconstruction was performed following established protocols (Fig. 1) [3]. Using the NAC removal site (round shape) after SSM, a transversely laid fusiform excision

\section{Fig. 1. Skin sparing mastectomy}

Nipple-sparing mastectomy during immediate breast reconstruction is aesthetically advantageous, since the nipple-areolar complex is preserved. When this is not possible, a skin flap should be preserved through skin-sparing mastectomy, as shown in the figure.

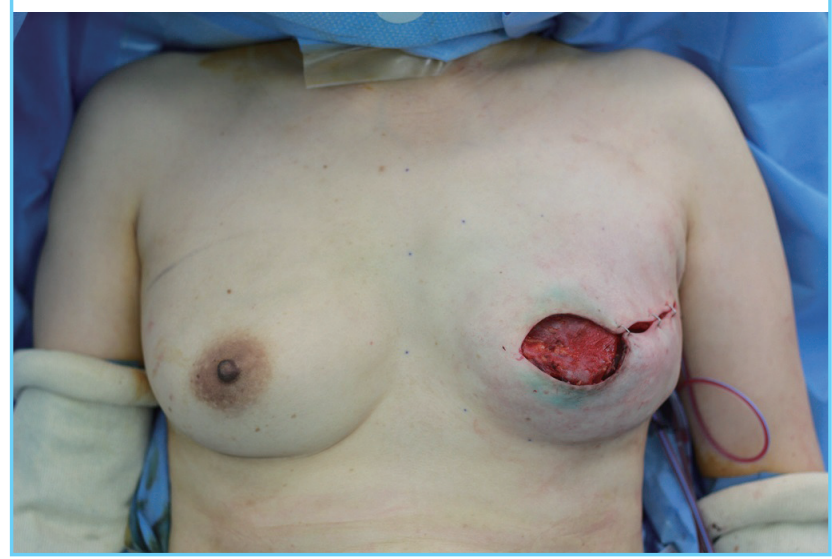

design was drawn, on which the long axis was 3 times longer than the NAC diameter. The long axis line and excision area were marked (Fig. 2). As shown in Fig. 3, the skin area to be ex-

\section{Fig. 2. Marking of the lazy $\mathrm{S}$ design}

Using the nipple-areolar complex (NAC) removal site (round shape) after skin-sparing mastectomy, a transversely laid fusiform excision design was drawn, on which the long axis was 3 times longer than the NAC diameter. The long axis line and excision area were marked.

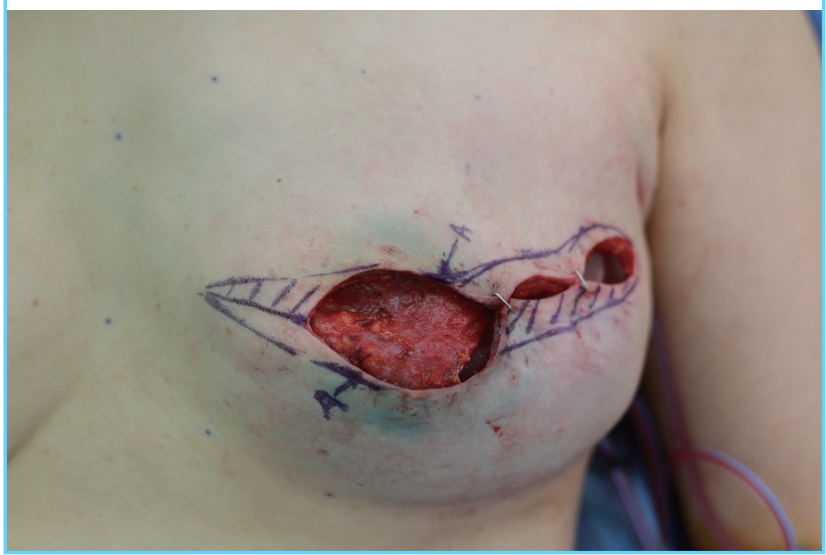

\section{Fig. 3. Selection of the skin excision area}

The skin area to be excised was selected based on 2 criteria for bilateral breast symmetry. (A) If the medial fullness of the contralateral normal breast was adequate, the lower medial and upper lateral skin area was selected for excision. (B) If the medial fullness of the contralateral normal breast was inadequate, the lower lateral and upper medial skin area was selected for excision.
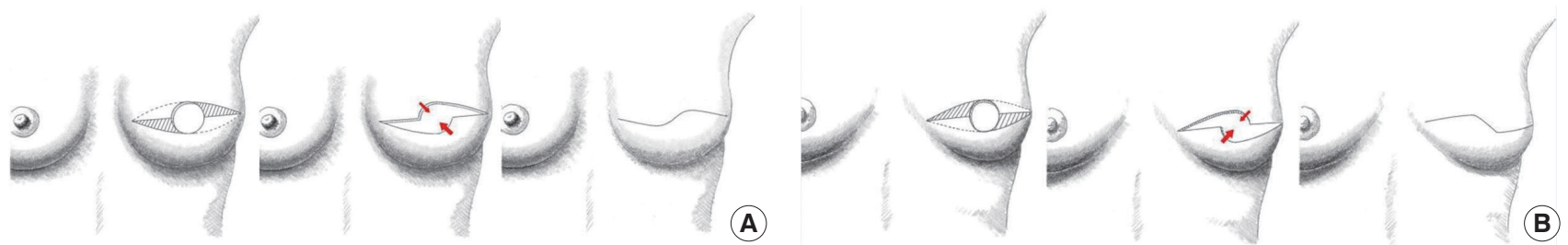

\section{Fig. 4. Immediate postoperative status after skin closure}

$(A, B)$ This patient underwent direct-to-implant breast reconstruction. These photographs show the patient's immediate postoperative status after skin closure using the lazy $\mathbf{S}$ design. The symmetry of both breast contours was well maintained (red arrows). The shape of the lazy $\mathbf{S}$ design breaks the straight line into 3 or more different directions and vectors, which may reduce the tension on the wound.
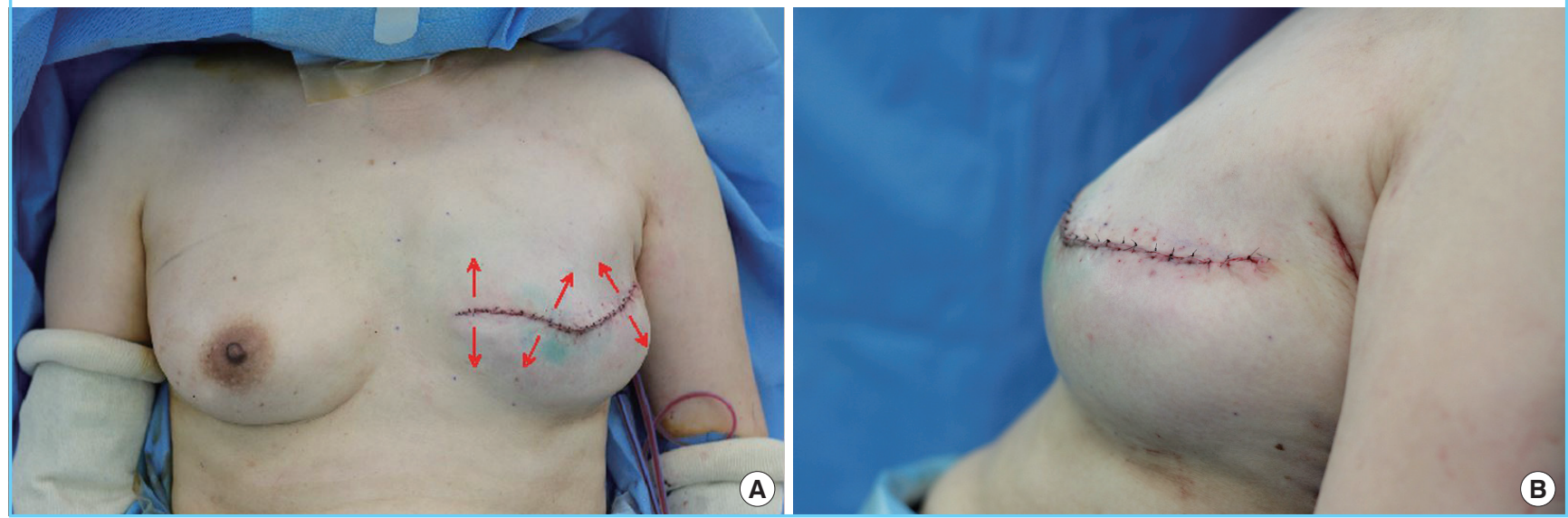


\section{Fig. 5. Postoperative photograph at 8 months}

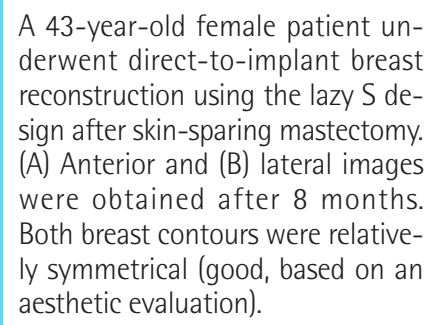

A 43-year-old female patient underwent direct-to-implant breast reconstruction using the lazy $S$ design after skin-sparing mastectomy. (A) Anterior and (B) lateral images were obtained after 8 months. Both breast contours were relatively symmetrical (good, based on an aesthetic evaluation).

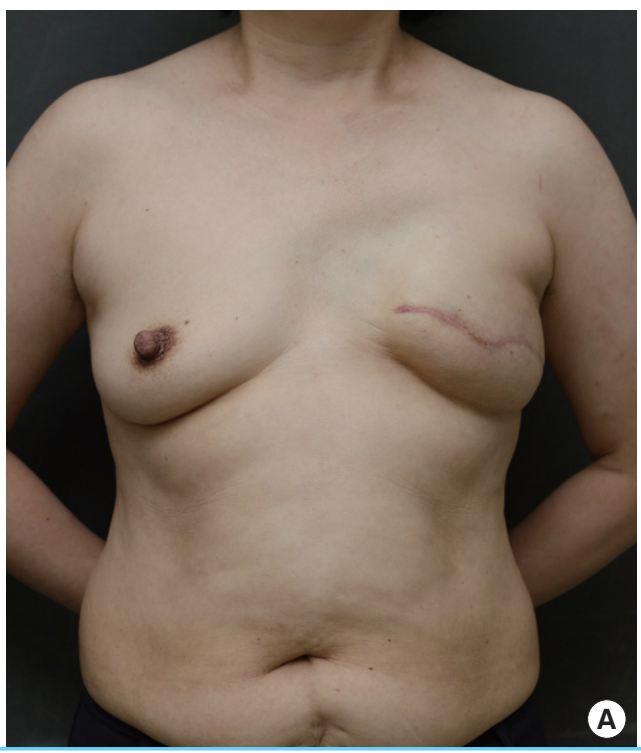

cised was selected based on 2 criteria for bilateral breast symmetry. If the medial fullness of the contralateral normal breast was adequate, the lower medial and upper lateral skin area was selected for excision (Fig. 3A). If the medial fullness of the contralateral normal breast was inadequate, the lower lateral and upper medial skin area was selected for excision (Fig. 3B). Skin closure was performed after tissue excision (Fig. 4).

We applied the lazy $S$ design to 14 subjects (14 breasts) who underwent immediate implant-based breast reconstruction after undergoing SSM for breast cancer between July 2014 and August 2015. DTI breast reconstruction was performed in 5 patients who wanted no additional surgery despite the possibility of a breast contour asymmetry. Nine patients underwent tissue expander-based breast reconstruction. The study was approved by the Institutional Review Board of the Kangbuk Samsung Hospital.

To assess the aesthetic outcomes, photographic analysis was performed 6 months after the final operation. With the lazy $S$ design, we were able to improve aesthetic outcomes. Fig. 5 presents a 43-year-old patient who showed relatively well-maintained breast symmetry at 8 months postoperatively.

\section{DISCUSSION}

Fusiform excision is the main technique used for closing round defects at the NAC site. However, in our experience with some cases, when it was used for implant-based breast reconstruction of round defects of the NAC caused by SSM, ptosis could not be replicated properly due to the lack of skin flap coverage. The lazy $S$ design may decrease by approximately $50 \%$ the amount of skin excised adjacent to the NAC defect.

Some attempts have been made to obtain more aesthetically pleasant results and to reduce wound complications in implantbased breast reconstruction. Dutton et al. [4] proposed a sinusoidal incision design on the lateral side of the NAC. This would produce a smaller scar and fewer wound complications due to the smaller incision length and the curved shape. Salgarello et al. [5] reported that a vertical excision design in implant-based breast reconstruction produced fewer wound complications than a wide skin excision design in women with large ptotic breasts. However, these designs did not reduce the amount of skin flap excision, unlike our design.

The advantage of decreasing the amount of skin excision is aesthetic. If the diameter of the NAC is $4 \mathrm{~cm}$ [6], with fusiform excision, $4 \mathrm{~cm}$ of the lower pole skin is pulled upward. However, with the lazy S design, approximately $50 \%$ of the skin is preserved and only $2 \mathrm{~cm}$ is pulled upwards, which is beneficial for symmetry. Fusiform excision can cause flattening of the central breast mound in many cases [1]. The lazy S design can supply skin to the center by oblique mobilization, which allows a more aesthetically appealing reconstruction.

The lazy $S$ design may reduce the tension on the wound by using 2 principles: less skin excision and conversion of the tension vectors. The shape of the lazy $S$ design breaks the straight line into 3 or more different directions and vectors. The central area of the wound thus better tolerates tension and gravity (Fig. 4A).

The efficiency of the design could be changed based on the ratio between the breast and the NAC. Although variations between individuals exist, the nipple-areola-breast proportion is usually defined as 1:3:9 $[6,7]$. The efficiency increases when the 
breast is large and the nipple and areola are small. However, the efficiency decreases when the breast is small and the nipple and areola are large.

In conclusion, the lazy $S$ design may be helpful for closing round-shaped wounds after SSM.

\section{REFERENCES}

1. Carlson GW. Technical advances in skin sparing mastectomy. Int J Surg Oncol 2011;2011:396901.

2. Carlson GW, Bostwick J 3rd, Styblo TM, et al. Skin-sparing mastectomy: oncologic and reconstructive considerations. Ann Surg 1997;225:570-5.

3. Becker H, Lind JG 2nd. The use of synthetic mesh in recon- structive, revision, and cosmetic breast surgery. Aesthetic Plast Surg 2013;37:914-21.

4. Dutton W, Ghareeb PA, McClellan WT. The lazy lateral incision: an innovative approach to the skin-sparing mastectomy. WV Med J 2013;109:30-3.

5. Salgarello M, Visconti G, Barone-Adesi L, et al. Inverted-T skin-reducing mastectomy with immediate implant reconstruction using the submuscular-subfascial pocket. Plast Reconstr Surg 2012;130:31-41.

6. Sanuki J, Fukuma E, Uchida Y. Morphologic study of nippleareola complex in 600 breasts. Aesthetic Plast Surg 2009;33: 295-7.

7. Hauben DJ, Adler N, Silfen R, et al. Breast-areola-nipple proportion. Ann Plast Surg 2003;50:510-3. 